\title{
Nonlinear evolution of r-modes: the role of differential rotation
}

\author{
Paulo M. Sa* and Brigitte Tome甘 \\ Departamento de Física and Centro Multidisciplinar de Astrofísica - CENTRA, \\ F.C.T., Universidade do Algarve, Campus de Gambelas, 8005-139 Faro, Portugal
}

(Dated: November 15, 2004)

\begin{abstract}
Recent work has shown that differential rotation, producing large scale drifts of fluid elements along stellar latitudes, is an unavoidable feature of $r$-modes in the nonlinear theory. We investigate the role of this differential rotation in the evolution of the $l=2 r$-mode instability of a newly born, hot, rapidly rotating neutron star. It is shown that the amplitude of the $r$-mode saturates a few hundred seconds after the mode instability sets in. The saturation amplitude depends on the amount of differential rotation at the time the instability becomes active and can take values much smaller than unity. It is also shown that, independently of the saturation amplitude of the mode, the star spins down to rotation rates that are comparable to the inferred initial rotation rates of the fastest pulsars associated with supernova remnants. Finally, it is shown that, when the drift of fluid elements at the time the instability sets in is significant, most of the initial angular momentum of the star is transferred to the $r$-mode and, consequently, almost none is carried away by gravitational radiation.

PACS numbers: 04.40.Dg, 95.30.Lz, 97.10.Sj, 97.10.Kc
\end{abstract}

\section{INTRODUCTION}

The interest in $r$-modes, first studied more than twenty years ago 1], has increased dramatically when it was discovered [2], and afterwards confirmed more generally [3] , that $r$-modes are driven unstable by gravitational radiation reaction in perfect-fluid stars with arbitrary small rotation. Soon afterwards, it was shown [4, 5] that in a newly born, hot, rapidly rotating neutron star, bulk and shear viscosity do not suppress the $r$-mode instability for a wide range of relevant temperatures and angular velocities of the star. As a result, the neutron star could spin down to just a small fraction of its initial angular velocity, thus providing a possible explanation for the relatively small spin rates of young pulsars in supernova remnants [4, 5]. The gravitational waves emitted by the young neutron star during this spin-down phase could be detected by enhanced versions of laser interferometer detectors 6 . It was also suggested that in accreting neutron stars in low-mass x-ray binaries the gravitational-wave emission due to the $r$-mode instability could balance the spin-up torque due to accretion, thus limiting the maximum angular velocity of these stars to values consistent with observations [7, 8].

Of fundamental importance in judging the astrophysical relevance of the $r$-mode instability is the determination of the saturation amplitude of the mode. In recent years, several authors have addressed this issue, both analytically and numerically, taking into account different nonlinear effects 19, 10, 11, 12, 13, 14, 15, 16].

An approximate analytical expression for differential rotation induced by $r$-modes was first derived using the linearized fluid equations by expanding the velocity of

\footnotetext{
*Electronic address: pmsa@ualg.pt
}

${ }^{\dagger}$ Electronic address: btome@ualg.pt a fluid element located at a certain point in powers of the mode's amplitude, averaging over a gyration, and retaining only the lowest-order nonvanishing term [9, 10]. This differential rotation can then interact with the magnetic field of a newly born, hot, rapidly rotating neutron star, limiting the growth of the $r$-mode instability or, for strong magnetic fields, even preventing it from developing $[9,11]$.

The first numerical study of nonlinear $r$-modes was performed for a rapidly-rotating relativistic star without a gravitational radiation force [12]. These studies show there is no suppression of the mode, even when its initial amplitude is of order unity, and also confirm the existence of differential rotation induced by r-modes. In a subsequent numerical study of the nonlinear evolution of $r$-modes of a rapidly-rotating Newtonian star with a gravitational radiation force, it was shown that the amplitude of the mode grows to order unity before strong shocks near the stellar surface rapidly damp the mode 13, 14]. However, a more recent numerical simulation has found no evidence that the decay of the mode's amplitude is due to such shocks near the surface of the star; instead, the catastrophic decay of a $r$-mode's amplitude of order unity is due to a leaking of energy into other fluid modes, leading to a differentially rotating configuration [15].

Recently, the nonlinear coupling between stellar inertial modes has been analyzed, with the conclusion that $r$-modes may saturate at much lower values than previous investigations had revealed [16]. Despite this fact, it was found that the $r$-mode instability could still explain the spin clustering at the fast end of the spin distribution of neutron stars in low-mass x-ray binaries and that gravitational waves from newly born, hot, rapidly rotating neutron stars, as well as from old neutron stars in low-mass x-ray binaries, could be detected by enhanced versions of laser interferometer gravitational wave detectors $[16$. 
In this paper, we are concerned with the role of differential rotation in the evolution of the $r$-mode instability.

Recently, a nonlinear extension of the linear $r$-mode perturbation was found within the nonlinear theory up to second order in the mode's amplitude $\alpha$ in the case of a Newtonian, barotropic, perfect-fluid star rotating with constant angular velocity $\Omega$ [17]. This nonlinear extension describes differential rotation of pure kinematic nature that produces large scale drifts along stellar latitudes. This solution contains two separate pieces, one induced by first-order quantities and another determined by the choice of initial data. These two pieces cannot cancel each other, since one is stratified on cylinders and the other not. Thus, differential rotation is an unavoidable kinematic feature of $r$-modes.

The differential rotation found in Ref. [17] does contribute to the second-order physical angular momentum of the $r$-mode, implying that the phenomenological model proposed in Ref. [6] to study the evolution of the $r$-mode instability in newly born, hot, rapidly rotating neutron stars has to be modified so that the physical angular momentum of the perturbation includes, not only the canonical angular momentum, but also the contribution from second-order differential rotation. In this paper, we study such a modified model, arriving at the conclusion that differential rotation plays an important role in the evolution of the $r$-mode instability.

In section 1 we derive an analytical expression for the physical angular momentum of the r-mode perturbation, using the second-order solution of Ref. 17]. In section IIII the model for the evolution of the $r$-mode instability is presented and a system of nonlinear, coupled, differential equations for the mode's amplitude $\alpha$ and the star's angular velocity $\Omega$ is derived. In section IV the analytical solution of the above mentioned system of differential equations is presented, revealing that differential rotation does play an important role in the evolution of the $r$-mode instability. Finally, in section $\square$ we present the conclusions.

\section{PHYSICAL ANGULAR MOMENTUM OF THE $R$-MODE PERTURBATION}

For small-amplitude perturbations of slowly rotating, Newtonian, barotropic, perfect-fluid stars, the $r$-mode solution to the linearized Euler and continuity equations is, in spherical coordinates $(r, \theta, \phi)$, given by:

$$
\begin{aligned}
& \delta^{(1)} v^{r}=0, \\
& \delta^{(1)} v^{\theta}=\alpha \Omega C_{l} l\left(\frac{r}{R}\right)^{l-1} \sin ^{l-1} \theta \sin (l \phi+\omega t), \\
& \delta^{(1)} v^{\phi}=\alpha \Omega C_{l} l\left(\frac{r}{R}\right)^{l-1} \sin ^{l-2} \theta \cos \theta \cos (l \phi+\omega t),
\end{aligned}
$$

where $\delta^{(1)} v^{i}$ are the contravariant components of the first-order Eulerian change in velocity, $R$ and $\Omega$ are, respectively, the radius and the angular velocity of the un- perturbed star,

$$
\omega=-\Omega l+\frac{2 \Omega}{l+1}
$$

is the mode's angular frequency in an inertial frame, $\alpha$ is the mode's amplitude and $C_{l}$ is chosen to be

$$
C_{l}=(2 l-1) ! ! \sqrt{\frac{2 l+1}{2 \pi(2 l) ! l(l+1)}} .
$$

To the velocity field (11) corresponds a Lagrangian vector displacement $\xi^{(1) i}$,

$$
\begin{aligned}
\xi^{(1) r} & =0, \\
\xi^{(1) \theta} & =-\frac{1}{2} \alpha C_{l} l(l+1)\left(\frac{r}{R}\right)^{l-1} \sin ^{l-1} \theta \cos (l \phi+\omega t), \\
\xi^{(1) \phi} & =\frac{1}{2} \alpha C_{l} l(l+1)\left(\frac{r}{R}\right)^{l-1} \sin ^{l-2} \theta \cos \theta \sin (l \phi+\omega t),
\end{aligned}
$$

satisfying, at lowest order in $\Omega$, the equations

$$
\delta^{(1)} v^{i}=\partial_{t} \xi^{(1) i}+v^{k} \nabla_{k} \xi^{(1) i}-\xi^{(1) k} \nabla_{k} v^{i}
$$

and

$$
\nabla_{i}\left(\rho \xi^{(1) i}\right)=0,
$$

where $v^{i}=\Omega \delta_{\phi}^{i}$ and $\rho$ are, respectively, the fluid velocity and the mass density of the unperturbed star. The knowledge of the first-order vector displacement $\xi^{(1) i}$ is sufficient to compute the canonical angular momentum of the $r$-mode perturbation [18]; using Eq. (4), one obtains:

$$
\begin{aligned}
J_{c} & =-\int \rho \partial_{\phi} \xi^{(1) i}\left(\partial_{t} \xi_{i}^{(1)}+v^{k} \nabla_{k} \xi_{i}^{(1)}\right) d V \\
& =-\frac{1}{4} \alpha^{2} \Omega l(l+1) R^{2-2 l} \int_{0}^{R} \rho r^{2 l+2} d r .
\end{aligned}
$$

However, the canonical angular momentum $J_{c}$ is not the full physical angular momentum at second order $\delta^{(2)} J$, the difference being given by [18]

$$
\delta^{(2)} J-J_{c}=\frac{1}{\Omega} \int \rho v^{i} \Delta_{\xi}^{(2)} v_{i} d V,
$$

where $\Delta_{\xi}^{(2)} v_{i}$ is the second-order Lagrangian change in velocity

$$
\begin{aligned}
\Delta_{\xi}^{(2)} v_{i}= & \partial_{t} \xi^{(1) k} \nabla_{i} \xi_{k}^{(1)}+v^{k} \nabla_{k} \xi^{(1) m} \nabla_{i} \xi_{m}^{(1)} \\
& +\partial_{t} \xi_{i}^{(2)}+v^{k}\left(\nabla_{i} \xi_{k}^{(2)}+\nabla_{k} \xi_{i}^{(2)}\right),
\end{aligned}
$$

which, as opposed to the canonical angular momentum, also contains terms linear in the second-order Lagrangian displacement vector $\xi^{(2) a}$, in addition to terms quadratic 
in $\xi^{(1) a}$. Therefore, the computation of the physical angular momentum at second order requires the knowledge of $\xi^{(2) a}$.

In a recent paper [17], a nonlinear extension of the linear $r$-mode was found within the nonlinear theory up to second order in the mode's amplitude in the case of a slowly rotating, Newtonian, barotropic, perfect-fluid star:

$$
\begin{aligned}
\delta^{(2)} v^{r}= & 0 \\
\delta^{(2)} v^{\theta}= & 0 \\
\delta^{(2)} v^{\phi}= & \frac{1}{2} \alpha^{2} \Omega C_{l}^{2} l^{2}\left(l^{2}-1\right)\left(\frac{r}{R}\right)^{2 l-2} \sin ^{2 l-4} \theta \\
& +\alpha^{2} \Omega A r^{N-1} \sin ^{N-1} \theta
\end{aligned}
$$

where $A$ and $N$ are arbitrary constants fixed by initial data. This second-order solution represents differential rotation, producing large scale drifts of fluid elements along stellar latitudes. The first term on the right-hand side of Eq. (10c) is induced by first-order quantities, while the second term is determined by the choice of initial data. Since these two terms cannot cancel each other, differential rotation is an unavoidable feature of $r$-modes. Let us in the present work restrict ourselves to the case $N=2 l-1$. We also redefine $A$ to be

$$
A=\frac{1}{2} C_{l}^{2} l^{2}(l+1) R^{2-2 l} K,
$$

where $K$ is a constant fixed by the choice of initial data.

At lowest order in $\Omega$, the contravariant components of the second-order Lagrangian displacement vector $\xi^{(2) i}$, corresponding to the axisymmetric time-independent velocity field (10), are determined by the equations [17]

$$
\begin{aligned}
\delta^{(2)} v^{i}= & \partial_{t} \xi^{(2) i}+v^{k} \nabla_{k} \xi^{(2) i}-\xi^{(2) k} \nabla_{k} v^{i} \\
& -\xi^{(1) k} \nabla_{k} \delta^{(1)} v^{i} \\
\nabla_{k} \xi^{(2) k}= & \frac{1}{2} \nabla_{k} \xi^{(1) m} \nabla_{m} \xi^{(1) k}, \\
\xi^{(2) k} \nabla_{k} \rho= & -\frac{1}{2} \xi^{(1) k} \xi^{(1) m} \nabla_{k} \nabla_{m} \rho,
\end{aligned}
$$

which yield the solution [17]:

$$
\begin{aligned}
\xi^{(2) r}= & \frac{1}{16} \alpha^{2} C_{l}^{2} l^{2}(l+1)^{2} R\left(\frac{r}{R}\right)^{2 l-1} \\
& \times \sin ^{2 l-2} \theta\left(\sin ^{2} \theta-2\right), \\
\xi^{(2) \theta}= & \frac{1}{16} \alpha^{2} C_{l}^{2} l^{2}(l+1)^{2}\left(\frac{r}{R}\right)^{2 l-2} \\
& \times \sin ^{2 l-3} \theta \cos \theta\left(\sin ^{2} \theta+2 l-2\right), \\
\xi^{(2) \phi}= & \frac{1}{4} \alpha^{2} \Omega C_{l}^{2} l^{2}(l+1)(2 K+2 l-1)\left(\frac{r}{R}\right)^{2 l-2} \\
& \times \sin ^{2 l-2} \theta t+C(r, \theta),
\end{aligned}
$$

where $C$ is an arbitrary function of $r$ and $\theta$. In Eq. (12)(14), terms quadratic in first-order quantities give rise to double "frequency" terms of the type $\cos [2(l \phi+\omega t)]$ inducing a second-order solution corresponding to an oscillating response at a "frequency" twice that of the linear $r$-mode; however, in this article these double frequency oscillations will not be considered since they give a zero contribution to the second-order physical angular momentum of the $r$-mode perturbation. Using the above expressions for the second-order Lagrangian displacement vector $\xi^{(2) i}$, the physical angular momentum at second order is computed to be 17]:

$$
\begin{aligned}
\delta^{(2)} J= & \frac{1}{2} \alpha^{2} \Omega[2 K l+(l-1)(2 l+1)] R^{2-2 l} \\
& \times \int_{0}^{R} \rho r^{2 l+2} d r .
\end{aligned}
$$

As can be seen from Eqs. (7) and (16), the physical angular momentum of the $r$-mode perturbation is not equal, in general, to the canonical angular momentum. The former contains also a part linear in the secondorder Lagrangian change in velocity, which, as pointed out in Ref. [18], is related to conservation of circulation in the fluid. Since, as shown in Ref. [17, at second order $r$-modes do not conserve vorticity, it follows that, in general, the physical and canonical angular momentum do not coincide. However, specific choices of $K$ can be made such that the integral in Eq. (8) vanishes. Such a case $(K=-2$, for $l=2)$ was studied in Ref. [6] within a phenomenological model for the evolution of the $r$-mode instability. There is not, however, to our knowledge, a physical condition that forces $K$ to take such a particular value. Therefore, in this paper we study the evolution of the $r$-mode instability for arbitrary values of $K$.

\section{THE EVOLUTION MODEL}

In this paper, the simple model proposed in Ref. 6 to study the evolution of the $r$-mode perturbation in newly born, hot, rapidly rotating neutron stars is adopted with two modifications. First, the physical angular momentum of the $r$-mode perturbation is not taken to be just the canonical angular momentum as in Ref. [6]. Instead, we will use the full physical angular momentum (16), which, as already discussed in the previous section, because of the presence of differential rotation induced by $r$-modes, is different, in general, from the canonical angular momentum. A second modification, less significant, is related to the proposal of Ref. 19] to deduce the evolution equations for the mode's amplitude $\alpha$ and the star's angular velocity $\Omega$ just from angular momentum considerations.

Since the most unstable $r$-mode is the $l=2$ mode, we will focus in this paper our attention only on the evolution of this mode. We will also assume, as in Ref. [6], that the mass density $\rho$ and the pressure $p$ of the fluid are related by a polytropic equation of state $p=k \rho^{2}$, with $k$ such that $M=1.4 M_{\odot}$ and $R=12.53 \mathrm{~km}$. 
In our model, it is assumed that the total angular momentum of the star is given by

$$
J=I \Omega+\delta^{(2)} J
$$

where the momentum of inertia of the equilibrium configuration $I$ is given by

$$
I=\frac{8 \pi}{3} \int_{0}^{R} \rho r^{4} d r=\tilde{I} M R^{2}
$$

with $\tilde{I}=0.261$, and the physical angular momentum of the $r$-mode perturbation $\delta^{(2)} J$ is given by

$$
\begin{aligned}
\delta^{(2)} J & =\frac{1}{2} \alpha^{2} \Omega(4 K+5) \frac{1}{R^{2}} \int_{0}^{R} \rho r^{6} d r \\
& =\frac{1}{2} \alpha^{2} \Omega(4 K+5) \tilde{J} M R^{2}
\end{aligned}
$$

with $\tilde{J}=1.635 \times 10^{-2}$.

The total angular momentum of the star $J=J(\alpha, \Omega)$ decreases due to the emission of gravitational radiation according to $[$ ]

$$
\frac{d J}{d t}=3 \tilde{J} M R^{2} \frac{\alpha^{2} \Omega}{\tau_{G R}},
$$

where the gravitational-radiation timescale $\tau_{G R}$ is given by 4

$$
\frac{1}{\tau_{G R}}=\frac{1}{\tilde{\tau}_{G R}}\left(\frac{\Omega^{2}}{\pi G \bar{\rho}}\right)^{3},
$$

with the fiducial timescale $\tilde{\tau}_{G R}=-3.26 \mathrm{~s}$. In the above equation, $\bar{\rho}$ is the average mass density of the star and $G$ is the gravitational constant.

From Eqs. (17) - 21) it is straightforward to obtain a differential equation for the time evolution of $\alpha$ and $\Omega$ :

$$
\begin{aligned}
{[1+} & \left.\frac{1}{3}(4 K+5) Q \alpha^{2}\right] \frac{d \Omega}{d t} \\
& +\frac{2}{3}(4 K+5) Q \Omega \alpha \frac{d \alpha}{d t}=2 Q \frac{\Omega \alpha^{2}}{\tau_{G R}}
\end{aligned}
$$

where $Q=3 \tilde{J} /(2 \tilde{I})=0.094$.

Following the proposal of Ref. [19] to deduce the evolution equations just from angular momentum considerations, we assume that the physical angular momentum of the $r$-mode perturbation $\delta^{(2)} J$ increases due to the emission of gravitational radiation and decreases due to the dissipative effect of viscosity,

$$
\frac{d \delta^{(2)} J}{d t}=-2 \delta^{(2)} J\left(\frac{1}{\tau_{G R}}+\frac{1}{\tau_{V}}\right) .
$$

For the viscous timescale $\tau_{V}$, we take the expression derived in Ref. 4] for the simple case of the linear $r$-mode (1) of a newly born, hot, rapidly rotating neutron star with shear and bulk viscosity:

$$
\frac{1}{\tau_{V}}=\frac{1}{\tilde{\tau}_{S}}\left(\frac{10^{9} \mathrm{~K}}{T}\right)^{2}+\frac{1}{\tilde{\tau}_{B}}\left(\frac{T}{10^{9} \mathrm{~K}}\right)^{6}\left(\frac{\Omega^{2}}{\pi G \bar{\rho}}\right),
$$

with the fiducial timescales $\tilde{\tau}_{S}=2.52 \times 10^{8} \mathrm{~s}$ and $\tilde{\tau}_{B}=$ $6.99 \times 10^{8} \mathrm{~s}$. Several authors have taken into account other dissipative mechanisms, but in this paper we shall restrict ourselves, for illustrative purposes, to the above expression.

From Eq. (23) one obtains, using Eq. (19), a second differential equation for the time evolution of $\alpha$ and $\Omega$ :

$$
2 \Omega \frac{d \alpha}{d t}+\alpha \frac{d \Omega}{d t}=-2 \alpha \Omega\left(\frac{1}{\tau_{G R}}+\frac{1}{\tau_{V}}\right) .
$$

Note that in Ref. [6] it was assumed that it was the energy of the perturbation (in the rotating frame), and not the angular momentum, that increases due to emission of gravitational waves and decreases due to viscosity. For a constant $\Omega$ these two approaches are coincident; however, for a varying $\Omega$ they differ in quantities of the order of $Q \alpha^{2}[19]$.

From Eqs. (22) and (25) it is straightforward to obtain a system of two, first-order, coupled, differential equations determining the time evolution of the amplitude of the $r$-mode $\alpha(t)$ and of the angular velocity of the star $\Omega(t)$ :

$$
\begin{aligned}
\frac{d \Omega}{d t}= & \frac{8}{3}(K+2) Q \frac{\Omega \alpha^{2}}{\tau_{G R}}+\frac{2}{3}(4 K+5) Q \frac{\Omega \alpha^{2}}{\tau_{V}}, \\
\frac{d \alpha}{d t}= & -\left[1+\frac{4}{3}(K+2) Q \alpha^{2}\right] \frac{\alpha}{\tau_{G R}} \\
& -\left[1+\frac{1}{3}(4 K+5) Q \alpha^{2}\right] \frac{\alpha}{\tau_{V}} .
\end{aligned}
$$

For $K=-2$, the above equations coincide with Eqs. (2.7) and (2.8) of Ref. [19] (for $1 / \tau_{M}=0$ ) and with Eqs. (3.14) and (3.15) of Ref. [6] (up to terms of order $\left.Q \alpha^{2}\right)$. In this paper, as already mentioned above, we study the evolution of the $r$-mode instability for an arbitrary value of $K$.

\section{THE ROLE OF DIFFERENTIAL ROTATION IN THE EVOLUTION OF $R$-MODES}

The condition $\tau_{G R}^{-1}(\Omega)+\tau_{V}^{-1}(\Omega, T)=0$ gives the stability curve, i.e., the set of points in a diagram $(\Omega, T)$ for which the damping effect of viscosity balances exactly the driving effect of gravitational radiation. For a newly born, hot, rapidly rotating neutron star, there is an interval of temperatures and angular velocities of the star for which the gravitational timescale $\tau_{G R}$ is much smaller than the viscous timescale $\tau_{V}$, implying that the evolution of $\alpha$ and $\Omega$ is determined, in a good approximation, by the equations:

$$
\begin{aligned}
& \frac{d \Omega}{d t}=\frac{8}{3}(K+2) Q \frac{\Omega \alpha^{2}}{\tau_{G R}}, \\
& \frac{d \alpha}{d t}=-\left[1+\frac{4}{3}(K+2) Q \alpha^{2}\right] \frac{\alpha}{\tau_{G R}} .
\end{aligned}
$$


These equations remain a good approximation to Eqs. (26) and (27) for a period of time during which the temperature and angular velocity of the neutron star are such that the corresponding point, in a $(\Omega, T)$ diagram, lies well above the stability curve. For the model we have been considering, this period of time is about one year [6].

In solving the above system of equations, initial conditions at $t=t_{0}$ are chosen such that $\left|\delta^{(2)} J\left(t_{0}\right)\right| \ll I \Omega\left(t_{0}\right)$. For $\alpha_{0} \equiv \alpha\left(t_{0}\right)=10^{-6}$, this implies that $|K| \ll 10^{13}$. In the case $K<-5 / 4$, for which $\delta^{(2)} J<0$, as the amplitude of the mode grows due to the gravitationalradiation instability, the total angular momentum of the star, given by Eq. (17), decreases and eventually becomes negative. To avoid this, the growth of the mode's amplitude has to be stopped by hand at a saturation value $\alpha_{\text {sat }} \leqslant \sqrt{-3 /[(4 K+5) Q]}$; integration is then carried on with a new set of equations for which $\alpha=\alpha_{\text {sat }}$ and the evolution of the angular velocity $\Omega$ is determined from Eq. (22), with $d \alpha / d t=0$,

$$
\frac{d \Omega}{d t}=\frac{2 \Omega}{\tau_{G R}} \frac{\alpha_{s a t}^{2} Q}{1+\frac{1}{3}(4 K+5) \alpha_{s a t}^{2} Q} .
$$

In the case $K \geqslant-5 / 4$, for which $\delta^{(2)} J \geqslant 0$, as the amplitude of the mode grows, the total angular momentum decreases but remains always positive and, therefore, there is no need to saturate the mode by hand. In this case, $\alpha(t)$ and $\Omega(t)$ are determined solely by Eqs. (28) and (29) and, as will be shown below, the mode saturates naturally at a value that depends on the parameter $K$, namely, $\alpha_{\text {sat }} \propto(K+2)^{-1 / 2}$. As already mentioned above, the parameter $K$, introduced in Eq. (11), is fixed by initial data, giving the initial amount of differential rotation associated to the $r$-mode. Thus, depending on the choice of initial data, the saturation amplitude of the $r$-mode can be of order unity, as assumed in the first models of evolution of the $r$-mode instability [6], or very small, as recent studies within the nonlinear theory seem to imply [16].

The requirement that the total angular momentum is always non-negative, implies that the right-hand side of Eq. (29) is always positive, i.e., $\alpha(t)$ increases in time for any value of $K$. It is also worth mentioning that, according to Eq. (28), for $K \neq-2, \Omega(t)$ evolves on a gravitational timescale, that is, a change in the background motion of the star occurs simultaneously to the growth of the mode due to the gravitational-wave instability. This result is not completely unexpected, since such kind of behavior has already been reported in the case of a toy model of a thin spherical shell of a rotating incompressible fluid [20].

Let us first consider the case $K \geqslant-5 / 4$. As already mentioned above, in this case the total angular momentum of the star is always positive and the amplitude of the mode $\alpha(t)$ and the angular velocity of the star $\Omega(t)$ are determined solely by the system of Eqs. (28) and (29), from which it is straightforward to obtain:

$$
\frac{d \Omega}{\Omega}=-\frac{\frac{8}{3}(K+2) Q \alpha}{1+\frac{4}{3}(K+2) Q \alpha^{2}} d \alpha .
$$

This differential equation yields the solution

$$
\frac{\Omega}{\Omega_{0}}=\frac{1+\frac{4}{3}(K+2) Q \alpha_{0}^{2}}{1+\frac{4}{3}(K+2) Q \alpha^{2}},
$$

where $\Omega_{0} \equiv \Omega\left(t_{0}\right)$ and $\alpha_{0} \equiv \alpha\left(t_{0}\right)$ are, respectively, the initial angular velocity of the star and the initial amplitude of the $r$-mode perturbation. Using the above expression to eliminate $\Omega$ from Eq. (29) and integrating, one obtains:

$$
\begin{aligned}
-\frac{1}{\tilde{\tau}_{G R}} & \left(\frac{\Omega_{0}}{\sqrt{\pi G \bar{\rho}}}\right)^{6}\left[1+\frac{4}{3}(K+2) Q \alpha_{0}^{2}\right]^{6}\left(t-t_{0}\right) \\
= & \ln \frac{\alpha}{\alpha_{0}}+\sum_{n=1}^{5} \frac{5 !}{2 n(5-n) ! n !}\left[\frac{4}{3}(K+2) Q\right]^{n} \\
& \times\left(\alpha^{2 n}-\alpha_{0}^{2 n}\right) .
\end{aligned}
$$

In the initial stages of the evolution of the $r$-mode instability, the right-hand side of Eq. (33) is dominated by the first term and $\alpha$ increases exponentially,

$$
\alpha(t) \simeq \alpha_{0} \exp \left\{0.027\left(\frac{\Omega_{0}}{\Omega_{K}}\right)^{6}\left(t-t_{0}\right)\right\},
$$

as expected; for later times, the right-hand side of Eq. (33) is dominated by the last term $(n=5)$ and $\alpha$ increases very slowly as

$$
\alpha(t) \simeq 2.48\left(\frac{\Omega_{0}}{\Omega_{K}}\right)^{3 / 5}\left(t-t_{0}\right)^{1 / 10} \frac{1}{\sqrt{K+2}} .
$$

In Eqs. (34) and (35), $t-t_{0}$ is given in seconds, $\Omega_{K}=$ $(2 / 3) \sqrt{\pi G \bar{\rho}}$ is the Keplerian angular velocity at which the star starts shedding mass at the equator, we have used for the gravitational timescale the value $\tilde{\tau}_{G R}=-3.26 \mathrm{~s}$, and we have assumed that $K$ and $\alpha_{0}$ are such that $4(K+2) Q \alpha_{0}^{2} / 3 \ll 1$. The smooth transition between the regimes (34) and (35) occurs for $t-t_{0} \simeq$ few $\times 10^{2}$ seconds.

The equation (33) describes the time evolution of the amplitude of the mode between the moment the gravitational-wave instability sets in and the moment viscosity effects become dominant and start damping the mode. It reveals that saturation of the $r$-mode occurs just a few hundred seconds after the beginning of the exponential growth of $\alpha$. Thus, within the model of evolution we are studying, which includes the nonlinear effect of the differential rotation induced by $r$-modes, the mode's amplitude saturates in a natural way (see Fig. 1).

The saturation value of the amplitude of the mode depends crucially on the parameter $K, \alpha_{\text {sat }} \propto(K+2)^{-1 / 2}$. 


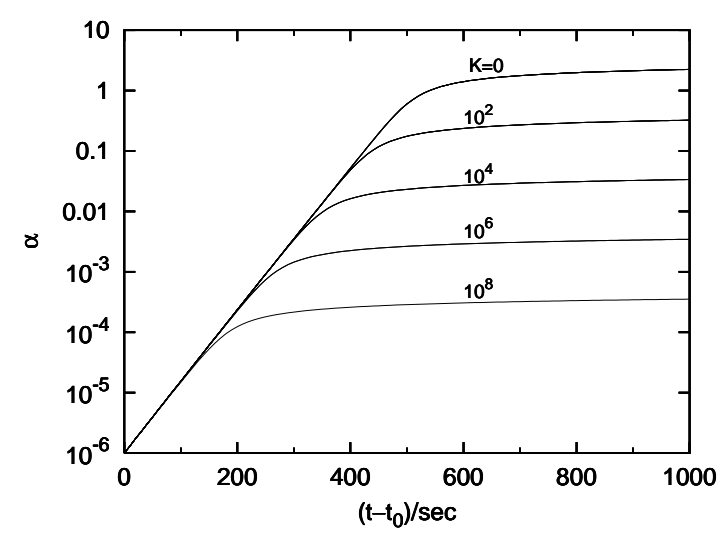

FIG. 1: Time evolution of the amplitude of the $r$-mode $\alpha$ for different values of $K$. The saturation value of the amplitude of the mode depends crucially on the parameter $K$, which is related to the amount of initial differential rotation associated with the $r$-mode. The initial values of the amplitude of the mode and of the angular velocity of the star are, respectively, $\alpha_{0}=10^{-6}$ and $\Omega_{0}=\Omega_{K}$.

The initial amount of differential rotation associated with the $r$-mode can be minimized by choosing $K$ appropriately, namely, $K \simeq 0$, with the consequence that the amplitude of the mode saturates at values of order unity. In this case, other nonlinear effects as, for instance, modemode interactions, that saturate $r$-modes at values much smaller than unity [16], are more relevant than differential rotation in what concerns the saturation amplitude of the mode. However, if the initial differential rotation associated to $r$-modes is significant, when compared with $\alpha_{0}^{-1}$, then the saturation amplitude $\alpha_{\text {sat }}$ can be as small as $10^{-3}-10^{-4}$. In this case, differential rotation plays an important role in the saturation of $r$-modes.

Let us now turn our attention to the time evolution of the angular velocity of the star $\Omega$. Integrating Eq. (28), after eliminating $\alpha$ with Eq. (32), one obtains the following solution:

$$
\begin{aligned}
-\frac{2}{\tilde{\tau}_{G R}}( & \left.\frac{\Omega_{0}}{\sqrt{\pi G \bar{\rho}}}\right)^{6}\left[1+\frac{4}{3}(K+2) Q \alpha_{0}^{2}\right]^{6}\left(t-t_{0}\right) \\
= & \sum_{n=1}^{5} \frac{\left[1+\frac{4}{3}(K+2) Q \alpha_{0}^{2}\right]^{n}}{n}\left[\left(\frac{\Omega_{0}}{\Omega}\right)^{n}-1\right] \\
& +\ln \frac{\Omega_{0}}{\Omega}+\ln \frac{1+\frac{4}{3}(K+2) Q \alpha_{0}^{2}-\frac{\Omega}{\Omega_{0}}}{\frac{4}{3}(K+2) Q \alpha_{0}^{2}} .
\end{aligned}
$$

In the initial stages of the evolution of the $r$-mode instability, the right-hand side of Eq. (36) is dominated by the last term and $\Omega$ decreases as

$$
\frac{\Omega(t)}{\Omega_{0}} \simeq 1-\frac{4}{3}(K+2) Q \alpha_{0}^{2} \exp \left\{0.054\left(\frac{\Omega_{0}}{\Omega_{K}}\right)^{6}\left(t-t_{0}\right)\right\}
$$

for later times, the right-hand side of Eq. (36) is dominated by the first term $(n=5)$ and $\Omega$ decreases slowly as

$$
\frac{\Omega(t)}{\Omega_{0}} \simeq 1.30\left(\frac{\Omega_{0}}{\Omega_{K}}\right)^{-6 / 5}\left(t-t_{0}\right)^{-1 / 5} .
$$

In Eqs. (37) and (38), again, $t-t_{0}$ is given in seconds, we have used for the gravitational timescale the value $\tilde{\tau}_{G R}=$ $-3.26 \mathrm{~s}$, and we have assumed that $K$ and $\alpha_{0}$ are such that $4(K+2) Q \alpha_{0}^{2} / 3 \ll 1$. The smooth transition between the regimes (37) and (38) occurs for $t-t_{0} \simeq$ few $\times 10^{2}$ seconds (see Fig. 2). Remarkably, in the later phase of

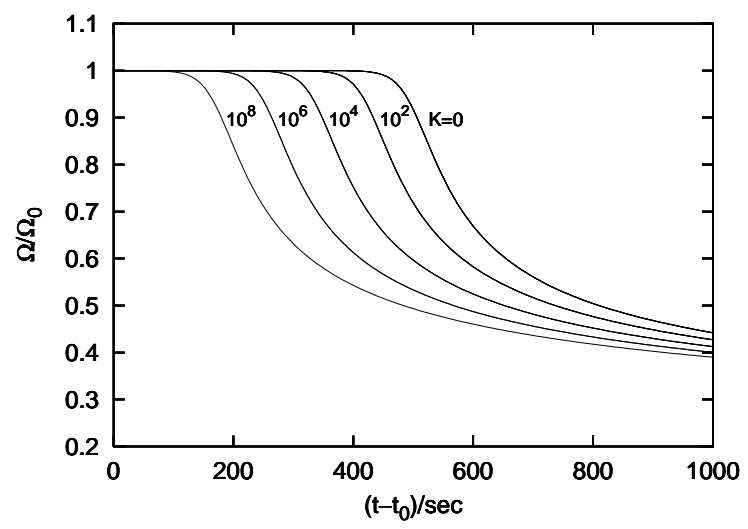

FIG. 2: Time evolution of the angular velocity of the star $\Omega$ for different values of $K$. After a few hundred seconds, the value of the angular velocity becomes quite insensitive to the value of $K$. The initial values of the amplitude of the mode and of the angular velocity of the star are, respectively, $\alpha_{0}=10^{-6}$ and $\Omega_{0}=\Omega_{K}$.

the evolution, the angular velocity $\Omega$ does not depend on the value of $K$, and, consequently, does not depend on the saturation value of $\alpha$. As can be seen in Fig. 2 already at $t-t_{0}=1000 \mathrm{~s}$, for values of $K$ ranging from 0 to $10^{8}$, the angular velocities are not much different; for $t-t_{0} \simeq 1$ year any difference becomes negligibly small. This contrasts with the results obtained in Ref. [] , where the value of $\Omega$ after about one year depends critically on the choice of $\alpha_{\text {sat }}$ (see case $K=-2$ below).

After about one year of evolution, when the dissipative effect of viscosity becomes dominant and starts damping the mode, the angular velocity of the star reaches values consistent with observational results. Indeed, assuming that initially the star rotates with the maximum allowed angular velocity, $\Omega_{0}=\Omega_{K}=(2 / 3) \sqrt{\pi G \bar{\rho}}$, one obtains from Eq. (36) that $\Omega_{\text {one year }} \simeq 0.042 \Omega_{K}$, in good agreement with the inferred initial angular velocity of the fastest pulsars associated with supernova remnants.

From Eqs. (35) and (38) follows that, for $t \gg$ few $\times 10^{2}$ seconds, the quantity $\left(\Omega / \Omega_{0}\right) \alpha^{2}$ does not depend on time, just on $K$, namely, $\left(\Omega / \Omega_{0}\right) \alpha^{2} \simeq 8 /(K+2)$, implying that the fraction of the initial angular momentum of the star that is transferred to the $r$-mode is just a function of $K$,

$$
\delta^{(2)} J / J_{0} \simeq \frac{K+5 / 4}{K+2}, \quad \text { for } t \gg \text { few } \times 10^{2} \text { seconds }
$$


where we have taken $J_{0} \simeq I \Omega_{0}$. Thus, for $K \gg 1$, most of the initial angular momentum of the star $I \Omega_{0}$ is transferred to the $r$-mode perturbation and, consequently, almost none is carried away by gravitational radiation emission. On the other hand, for $K=-5 / 4$, the angular momentum of the perturbation $\delta^{(2)} J$ remains zero during the evolution and, consequently, all the initial angular momentum of the star $I \Omega_{0}$ is available to be radiated away by gravitational radiation (see Figs. 3 and 4 ).

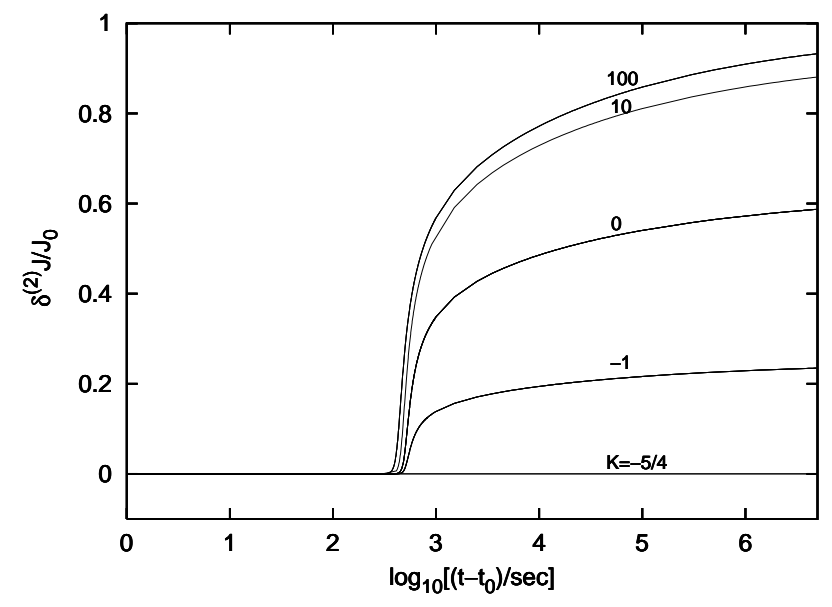

FIG. 3: Time evolution of the physical angular momentum of the $r$-mode perturbation $\delta^{(2)} J$ for different values of $K$. For $K \gg 1$, most of the initial angular momentum of the star $J_{0} \simeq I \Omega_{0}$ is transferred to the $r$-mode perturbation and, consequently, almost none is carried away by gravitational radiation emission. The initial values of the amplitude of the mode and of the angular velocity of the star are, respectively, $\alpha_{0}=10^{-6}$ and $\Omega_{0}=\Omega_{K}$.

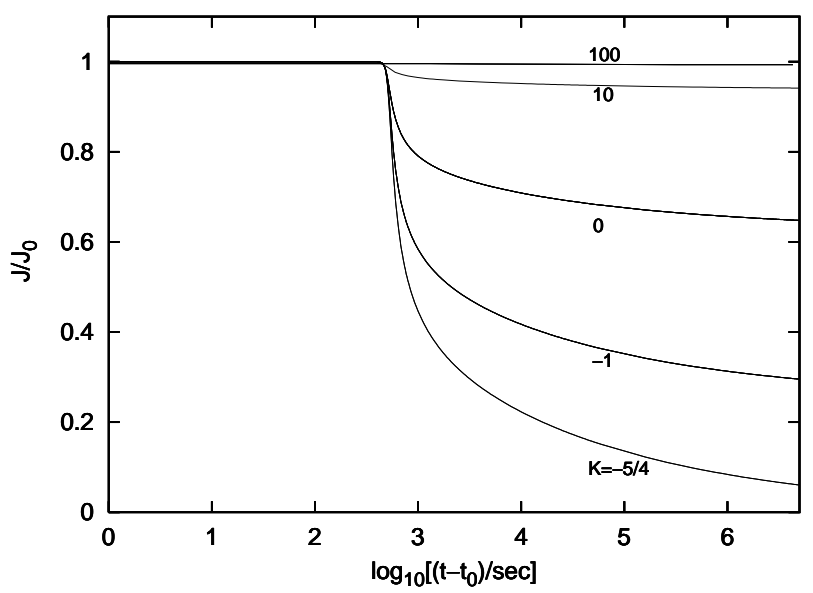

FIG. 4: Time evolution of the total angular momentum of the star $J$ for different values of $K$. For small values of $K$, a significant part of the initial angular momentum of the star is radiated away by gravitational radiation. The initial values of the amplitude of the mode and of the angular velocity of the star are, respectively, $\alpha_{0}=10^{-6}$ and $\Omega_{0}=\Omega_{K}$.

During the nonlinear evolution, the fluid develops a strong differential rotation. Let us define the average dif- ferential rotation $\Delta \Omega$ as the weighted variance of $\Omega[14]$,

$$
(\Delta \Omega)^{2}=\frac{\int \rho r^{2} \sin ^{2} \theta\left(\delta^{(2)} v^{\phi}-\bar{\Omega}_{d r}\right)^{2} d V}{\int \rho r^{2} \sin ^{2} \theta d V}
$$

where the average angular velocity $\bar{\Omega}_{d r}$, characterizing the drift of fluid elements along stellar latitudes, is given by

$$
\bar{\Omega}_{d r}=\frac{\delta^{(2)} J}{I}=\frac{\int \rho r^{2} \sin ^{2} \theta \delta^{(2)} v^{\phi} d V}{\int \rho r^{2} \sin ^{2} \theta d V} .
$$

For the polytropic model we have been considering, Eqs. (40) and (41) yield for the average differential rotation the following expression:

$$
\begin{aligned}
\Delta \Omega= & \frac{1}{3} \alpha^{2} \Omega Q\left[\frac{15}{56}\left(24 K^{2}+56 K+35\right) \frac{\tilde{I} \tilde{H}}{\tilde{J}^{2}}\right. \\
& \left.-(4 K+5)^{2}\right]^{1 / 2},
\end{aligned}
$$

where $\tilde{H}=\int_{0}^{R} \rho(r) r^{8} d r /\left(M R^{6}\right)=0.01$. As can be seen from Fig. 5$]$ after a few hundred seconds the average differential rotation increases rapidly, saturating at high values relatively to the initial angular velocity of the star. Note that the average differential rotation never vanishes

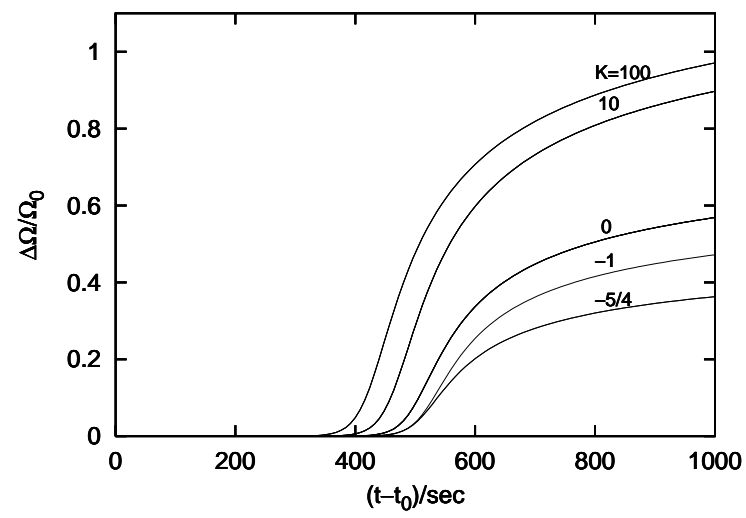

FIG. 5: Time evolution of the average differential rotation $\Delta \Omega$ for different values of $K$. After a few hundred seconds the average differential rotation increases rapidly, saturating at high values relatively to the initial angular velocity of the star. The initial value of the amplitude of the mode is $\alpha_{0}=10^{-6}$.

for any value of $K$. This is a consequence of the fact that the second-order velocity field (10) has two components, one induced by first-order quantities and another, fixed by initial data, which is a pure second-order effect. Since these two terms cannot cancel each other, a velocity drift of fluid elements along stellar latitudes is an unavoidable feature of the nonlinear $r$-mode pulsation.

Let us now turn to the case $K<-5 / 4$. As already mentioned above, at a certain point of the evolution, the $r$-mode has to be saturated by hand in order to avoid 
that the total angular momentum of the star becomes negative. Thus, during a first stage of the evolution, $\alpha$ and $\Omega$ are determined from Eqs. (28) and (29) and during a second stage, $\alpha=\alpha_{\text {sat }} \leqslant \sqrt{-3 /[(4 K+5) Q]}$ and $\Omega$ is determined by Eq. (30). The fact that a saturation value for $\alpha$ has to be fixed by hand introduces an element of arbitrariness which did not exist in the case $K \geqslant-5 / 4$. In particular, by choosing appropriately $\alpha_{\text {sat }}$ it is possible to arrange that the final value of $\Omega$ is consistent with observational data and, additionally, that a significant part of the initial angular momentum of the star is carried away by gravitational waves.

For $-2<K<-5 / 4$ and $K<-2$, the solution of the system of equations (28) and (29) is given by Eqs. (33) and (36). During the second stage of evolution, $\alpha=$ $\alpha_{\text {sat }} \leqslant \sqrt{-3 /[(4 K+5) Q]}$ and $\Omega(t)$ is given by

$$
\begin{aligned}
\Omega(t)= & \Omega\left(t_{*}\right)\left[\frac{0.030 \alpha_{\text {sat }}^{2}}{1+\frac{1}{3}(4 K+5) Q \alpha_{\text {sat }}^{2}}\right. \\
& \left.\times\left(\frac{\Omega\left(t_{*}\right)}{\Omega_{K}}\right)^{6}\left(t-t_{*}\right)+1\right]^{-1 / 6},
\end{aligned}
$$

where $t_{*}$ is the time at which occurs the transition from the first to the second stage of evolution and $t-t_{*}$ is given in seconds. During the two stages of evolution we have just described, the total angular momentum of the star $J(t)$ decreases due to gravitational-wave emission. At the end of the second stage of the evolution, it is given by

$$
J\left(t_{\text {final }}\right) \simeq J_{0}\left[1+\frac{1}{3}(4 K+5) Q \alpha_{\text {sat }}^{2}\right] \frac{\Omega\left(t_{\text {final }}\right)}{\Omega_{0}}
$$

where we have taken $J_{0} \simeq I \Omega_{0}$ and $\Omega(t)$ is given by Eq. (43). Finally, let us point out that, for $K<-2$, during the first stage of evolution, the angular velocity of the star increases.

The case $K=-2$ has to be treated separately. It corresponds to the model studied in detail in Ref. 6]. The system of equations (28) and (29) yields the solution $\Omega=$ $\Omega_{0}$ and $\alpha=\alpha_{0} \exp \left\{-\left(t-t_{0}\right) / \tau_{G R}\right\}$. If the initial angular velocity is chosen to be $\Omega_{0}=\Omega_{K}$, then $\tau_{G R}=-37.1 \mathrm{~s}$, implying that the perturbation grows exponentially from the initial amplitude $\alpha_{0}=10^{-6}$ to values of the order unity in just about $500 \mathrm{~s}$ 6]. After this short initial period in which $\Omega$ is constant and $\alpha$ grows exponentially, the amplitude $\alpha$ has to be forced, by hand, to take a certain saturation value $\alpha_{\text {sat }} \leqslant Q^{-1 / 2}=3.26$, and the angular velocity of the star is then given by Eq. (43), with $K=$ -2 and $\Omega\left(t_{*}\right)=\Omega_{0}$. The final angular momentum of the star is given by Eq. (44) with $K=-2$. As can be seen from these equations, the final angular velocity and momentum of the star depend critically on the saturation value of the mode's amplitude $\alpha_{\text {sat }}$; for instance, after one year of evolution, for $\alpha_{\text {sat }}=1$ and $\Omega_{0}=\Omega_{K}$ one obtains $\Omega \simeq 0.1 \Omega_{K}$ and $J \simeq 0.09 J_{0}$, while for $\alpha_{\text {sat }}=10^{-3}$ the angular velocity and momentum are, respectively, $\Omega \simeq$ $0.9 \Omega_{K}$ and $J \simeq 0.9 J_{0}$.

\section{CONCLUSIONS}

In this paper we have studied the role of differential rotation in the evolution of the $r$-mode instability. We have adopted the simple model of Ref. [6], with two modifications: (i) the physical angular momentum of the $r$ mode perturbation includes not only the canonical angular momentum but also a piece linear in second-order quantities, corresponding to differential rotation inducing large scale drifts of fluid elements along stellar latitudes; (ii) the evolution equations are deduced just from angular momentum considerations. The first modification is a quite important one, resulting from the fact that differential rotation is an unavoidable kinematic feature of $r$-modes [17]. The presence of this differential rotation implies that at second order in the mode's amplitude $r$ modes do not preserve vorticity of fluid elements. This in turn implies that the canonical angular momentum is not the full angular momentum at second order; one should also include a part linear in the second-order Lagrangian change in velocity, which is related to conservation of circulation in the fluid. The second modification, less significant, leads to a system of equations for the evolution of the $r$-mode instability that differs from the ones of Ref. [6] just in a quantity of the order of $Q \alpha^{2}$. A detailed justification for this modification can be found in Ref. [19].

Within this model, we have derived a system of two first-order, coupled, differential equations (26) and (27), determining the time evolution of the amplitude of the $r$-mode $\alpha(t)$ and the angular velocity of the star $\Omega(t)$. For the gravitational and viscous timescales appearing in these equations, we have used the expressions derived in Refs. [4, 5] for the simple case of $r$-modes of a newly born, hot, rapidly rotating neutron star with shear and bulk viscosity. In this case, the driving effect of the gravitational radiation reaction overcomes the damping effect of shear and bulk viscosity for about one year, while the temperature of the star decreases from about $10^{10} \mathrm{~K}$ to about $10^{9} \mathrm{~K}$. During this period of time, in which the $r$ mode instability is active, the gravitational timescale is much smaller than the viscous timescale, $\tau_{G R} \ll \tau_{V}$, and, therefore, the evolution of $\alpha$ and $\Omega$ can be determined, in a good approximation, by the system of equations (28) and (29).

The system of equations (28) and (29) contains a parameter $K$, which is fixed by initial data and gives the initial amount of differential rotation associated with the $r$-mode. The specific case $K=-2$, for which the physical angular momentum of the $r$-mode perturbation coincides with the canonical angular momentum, was studied in great detail in Ref. [6]. There is not, however, to our knowledge, any physical condition that forces $K$ to take such a particular value $K=-2$. Therefore, we have solved, both numerically and analytically, the system of equations (28) and (29) for arbitrary $|K| \ll 10^{13}$. This upper limit for $K$ results from the fact that one wishes to impose the condition that the initial absolute value 
of the physical angular momentum of the perturbation $\left|\delta^{(2)} J\left(t_{0}\right)\right|$ is much smaller than the angular momentum of the unperturbed star $I \Omega_{0}$. In terms of the initial drift of fluid elements along stellar latitudes, this condition implies that $\left(v_{d r}\right)_{0} \ll \Omega_{0} R$ at the equator of the star. It could be argued that some dissipative mechanism, such as bulk viscosity or magnetic coupling, would reduce differential rotation in a newly born, hot, rapidly rotating neutron star, before the $r$-mode instability sets in, implying that $K \simeq 0$. However, even in that case, a residual differential rotation of $r$-modes would be present in the star and the average differential rotation $\Delta \Omega$ would increase exponentially as the amplitude of the $r$-mode grows, saturating at values relatively high as compared with the initial angular velocity of the star. Finally, in what concerns the admissible values of $K$, let us point out that for $K<-5 / 4$, as the amplitude of the mode grows due to the gravitational-radiation instability, the total angular momentum of the star decreases and eventually becomes negative. To avoid this unphysical situation, the growth of the mode's amplitude has to be stopped by hand at a saturation value $\alpha_{\text {sat }} \leqslant \sqrt{-3 /[(4 K+5) Q]}$ and integration has then to be carried on with a new set of equations. The fact that a saturation value for $\alpha$ has to be fixed by hand introduces an element of arbitrariness into the solution, permitting, for instance, that agreement between the predicted final value of the angular velocity of the star and the value inferred from astronomical observations can always be achieved by simply fine tuning the value of $\alpha_{\text {sat }}$. One would wish, of course, that such an agreement, even qualitative, would arise in a natural way, without fine tuning the parameters of the model. For this reason, we have concentrated most of our attention on the case $K \geqslant-5 / 4$, in which such arbitrariness does not exist.

From the exact analytical solution (33) and (36) of the system of equations (28) and (29) one can extract several conclusions.

First, the amplitude of the $r$-mode saturates in a natural way a few hundred seconds after the mode instability sets in. The saturation amplitude depends on the parameter $K$, namely, $\alpha_{\text {sat }} \propto(K+2)^{-1 / 2}$. Therefore, if the initial differential rotation of $r$-modes is small $(K \simeq 0)$, then the $r$-mode saturates at values of the order of unity. On the other hand, if the initial differential rotation is significant $(K \gg 1)$, then the saturation amplitude can be as small as $10^{-3}-10^{-4}$. These low values for the saturation amplitude of $r$-modes are of the same order of magnitude as the ones obtained in recent investigations on wind-up of magnetic fields $[9]$ and on nonlinear mode-mode interaction 16.

Second, the value of the angular velocity of the star becomes, after a short period of evolution $\left(t \gg\right.$ few $\times 10^{2}$ $\mathrm{s})$, very insensitive to the value of the parameter $K$, i.e., it becomes insensitive to the saturation value of the mode's amplitude. From Eq. (36) for $t=t(\Omega)$, it can be easily obtained that the angular velocity after about one year of evolution of the $r$-mode instability is $0.042 \Omega_{K}$ (for any $K$ ), in good agreement with the inferred initial angular velocity of the fastest pulsars associated with supernova remnants.

Finally, the value of the physical angular momentum $\delta^{(2)} J$ of the $r$-mode perturbation tends, after a short period of evolution $\left(t \gg\right.$ few $\left.\times 10^{2} \mathrm{~s}\right)$, to a constant value given approximately by $\left[\left(K+\frac{5}{4}\right) /(K+2)\right] J_{0}$. Thus, for $K \gg 1$ most of the initial angular momentum of the star is transferred to the $r$-mode and, consequently, almost none is carried away by gravitational radiation. That the $r$-mode absorbs most of the initial angular momentum of the star is explained by the fact that the mode develops a very strong differential rotation. Indeed, the average differential rotation, given by Eq. (42), increases exponentially, saturating after a few hundred seconds of evolution at very high values relatively to the initial angular velocity of the star. On the other hand, for values of $K \simeq-5 / 4$, the transfer of angular momentum to the $r$-mode is less strong and, consequently, more angular momentum is available to be carried away by gravitational radiation.

As we have seen, differential rotation introduces in the evolutionary picture of the $r$-mode instability new and somewhat unexpected features. This differential rotation has a kinematic origin, being induced by terms quadratic in the velocity field of the linear $r$-mode. However, differential rotation of $r$-modes could also be induced by the gravitational-radiation reaction force. Indeed, within a toy model of a thin spherical shell of a rotating incompressible fluid it was shown that differential rotation of $r$-modes is also driven by a gravitational radiation force, leading to the conjecture that in real stars one could observe a similar behavior [20]. The existence of this differential rotation induced by gravitational radiation and its influence on the evolution of the $r$-mode instability will be addressed in a future publication [21].

\section{Acknowledgments}

We thank Óscar Dias for helpful discussions. This work was supported in part by the Fundação para a Ciência e a Tecnologia (FCT), Portugal. BT acknowledges financial support from FCT through grant PRAXIS XXI/BD/21256/99.
[1] J. Papaloizou and J. E. Pringle, Mon. Not. R. Astron. Soc. 182, 423 (1978).

[2] N. Andersson, Astrophys. J. 502, 708 (1998).
[3] J. L. Friedman and S. M. Morsink, Astrophys. J. 502, 714 (1998).

[4] L. Lindblom, B. J. Owen, and S. M. Morsink, Phys. Rev. 
Lett. 80, 4843 (1998).

[5] N. Andersson, K. D. Kokkotas, and B. F. Schutz, Astrophys. J. 510, 846 (1999).

[6] B. J. Owen, L. Lindblom, C. Cutler, B. F. Schutz, A. Vecchio, and N. Andersson, Phys. Rev. D 58, 084020 (1998).

[7] L. Bildsten, Astrophys. J. 501, L89 (1998).

[8] N. Andersson, K. D. Kokkotas, and N. Stergioulas, Astrophys. J. 516, 307 (1999).

[9] L. Rezzolla, F. K. Lamb, and S. L. Shapiro, Astrophys. J. 531, L141 (2000).

[10] L. Rezzolla, F. K. Lamb, D. Markovic, and S. L. Shapiro, Phys. Rev. D 64, 104013 (2001).

[11] L. Rezzolla, F. K. Lamb, D. Markovic, and S. L. Shapiro, Phys. Rev. D 64, 104014 (2001).

[12] N. Stergioulas and J. A. Font, Phys. Rev. Lett. 86, 1148 (2001).

[13] L. Lindblom, J. E. Tohline, and M. Vallisneri, Phys. Rev.
Lett. 86, 1152 (2001).

[14] L. Lindblom, J. E. Tohline, and M. Vallisneri, Phys. Rev. D 65, 084039 (2002).

[15] P. Gressman, L.-M. Lin, W.-M. Suen, N. Stergioulas, and J. L. Friedman, Phys. Rev. D 66, 041303(R) (2002).

[16] P. Arras, E. E. Flanagan, S. M. Morsink, A. K. Schenk, S. A. Teukolsky, and I. Wasserman, Astrophys. J. 591, 1129 (2003).

[17] P. M. Sá, Phys. Rev. D 69, 084001 (2004).

[18] J. L. Friedman and B. F. Schutz, Astrophys. J. 221, 937 (1978).

[19] W. C. G. Ho and D. Lai, Astrophys. J. 543, 386 (2000).

[20] Yu. Levin and G. Ushomirsky, Mon. Not. R. Astron. Soc. 322, 515 (2001).

[21] O. J. C. Dias and P. M. Sá, in preparation. 